\title{
Evidence that Osteoblasts are Specialized Citrate-producing Cells that Provide the Citrate for Incorporation into the Structure of Bone
}

\author{
Renty B. Franklin ${ }^{1}$, Meena Chellaiah ${ }^{1}$, Jing Zou ${ }^{1}$, Mark A. Reynolds ${ }^{2}$ and Leslie C. Costello ${ }^{1, *}$ \\ ${ }^{1}$ Department of Oncology and Diagnostic Sciences; Dental School; University of Maryland, Baltimore, Md. 21201 \\ ${ }^{2}$ Department of Periodontics; Dental School; University of Maryland, Baltimore, Md. 21201
}

\begin{abstract}
Citrate is a major component of bone in all vertebrates, but its implications in bone have remained largely unknown. Recent studies identified that citrate is incorporated into the structure of the hydroxyapatite nanocrystal/collagen complex; and is essential for the important biomechanical properties of bone. This raises the important question, "What is the source of citrate for incorporation into bone?"; A question that heretofore had remained unresolved. Studies in this report were designed to determine the plausibility of our concept that the osteoblasts are specialized citrate-producing cells, which provide the citrate that is incorporated into the structure of bone; and that osteogenic differentiation of mesenchyme cells leads to the development of the citrate-producing osteoblasts. The results demonstrated that primary human osteoblasts exhibit the capability of citrate-production. Undifferentiated mesenchyme cells do not exhibit the capability of citrate production; and osteogenic differentiation results in citrate-producing osteoblasts. The up-regulation of zinc uptake transporter ZIP1 is essential for the manifestation of the citrate-producing capability of the osteoblasts. We determined that osteoblast transport of citrate from plasma is not a likely source of citrate in bone. Thus, this study establishes for the first time that the osteoblasts are specialized citrate-producing cells that provide the citrate for incorporation into the structure of bone; and that mesenchyme cell osteogenesis leads to differentiated citrate-producing osteoblasts. This is a new understanding; which must include the osteogenic development of citrate-producing osteoblasts, and the process of "citration" in concert with mineralization during bone formation. It also provides a new understanding of the role of bone in the homeostatic maintenance of plasma citrate concentration.
\end{abstract}

Keywords: Bone formation, citrate production, mesenchyme cells, osteoblasts, ZIP1 transporter, zinc.

\section{INTRODUCTION}

Since the initial study of Dickens in 1941 [1], numerous subsequent reports have established that bone contains extremely high levels of citrate (for review [2]). Bone (and dentine) citrate levels are generally $\sim 20-100 \mu$ mols/gram. For comparison with soft issues, this would translate to $\sim 5$ 25 umols/gram wet-weight for bone; which contrasts with 0.1-0.5 umol/gram wet-weight for most soft tissues. Thus bone contains $\sim 50$-fold more citrate than exists in other tissues (with some exception); and is even $\sim 10-20$ fold greater than cartilage [1]. Citrate comprises $\sim 1.6 \%$ of the bone content; $\sim 5 \%$ of the organic component of bone; and $\sim 80 \%$ of the total body citrate resides in bone. Moreover, the high citrate content of bone exists in all osteo-vertebrates as an evolution change from chondro-vertebrates. These relationships dictate that citrate is a major indispensible component of bone, which must be involved in some essential functional/structure role that is required for the development and maintenance of normal bone.

*Address correspondence to this author at the Department of Oncology and Diagnostic Sciences, Dental School; University of Maryland, 650 West Baltimore Street, Baltimore, Md. 21201; Tel: 410706 7618;

E-mail: 1costello@umaryland.edu
Despite this, the role of citrate in bone, its source, its regulation, and other critical issues have remained largely unresolved over the $\sim 75$ years since first identified as a major component of bone. Moreover, for the past $\sim 35$ years, the implications and even the existence of citrate in bone has been ignored by the clinical and biomedical research community; to the extent that it is not even described as a component of bone in recent textbooks and review articles $[3,4]$. Thus, aside from the interest and research of the early investigators, there has been minimal contemporary attention, research, and advances regarding the implications of citrate in normal bone and in bone disorders. However, new insight into the role of citrate has now been provided by the recent identification of citrate incorporation in the structure of the apatite nanocrystal/collagen complex structure [4-7]; and is essential for imparting the important biomechanical properties of bone (e.g. stability, strength, and resistance to fracture) This brings renewed attention to the importance of citrate in bone, and the need to address and resolve the issues described above.

The focus of this report is the important unresolved issue, "What is the source of citrate that is incorporated into the structure of bone?" Contrary to prevailing views that citrate in bone is derived from blood, we have proposed that the osteoblasts are functional metabolic "citrate-producing" cells that provide the citrate for incorporation into the structure of 


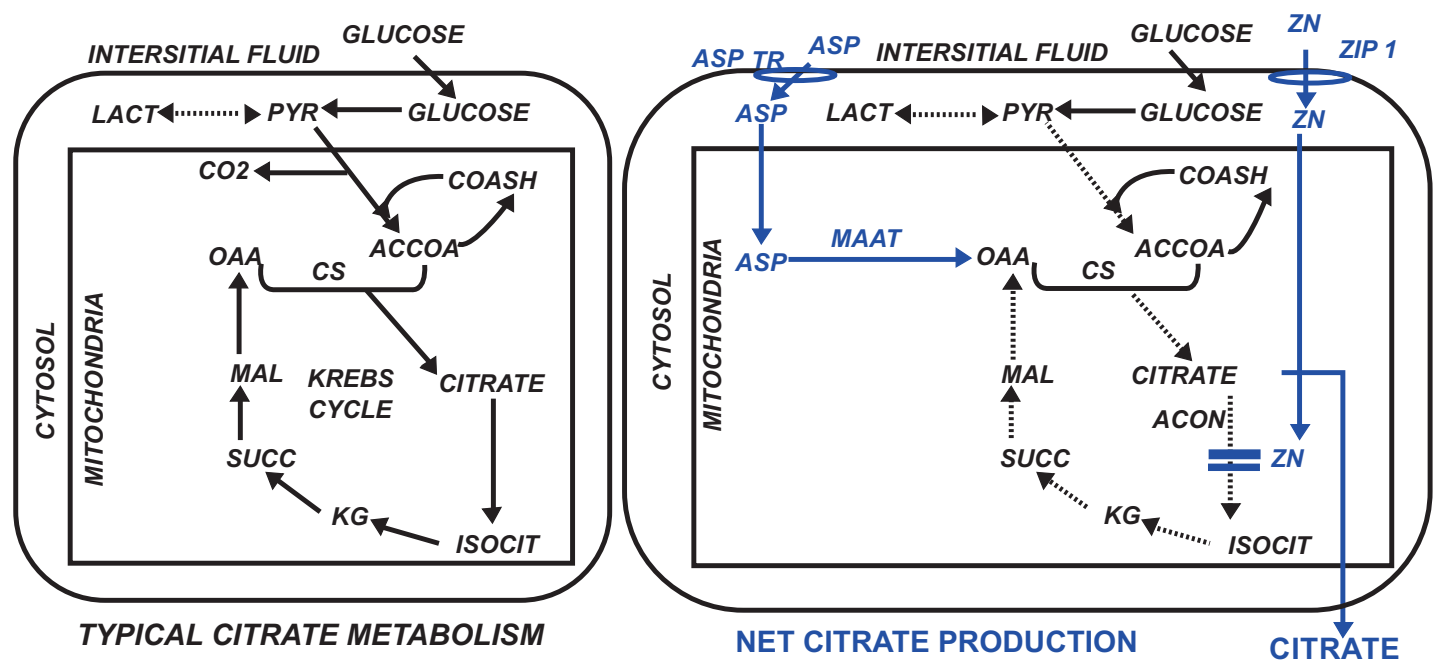

Fig. (1). Comparison of typical citrate metabolism in most mammalian cells versus net citrate production in citrate-producing cells. Blue highlights some major modifications associated with citrate production. $\mathrm{CS}=$ citrate synthase; Acon= m-aconitase.; MAAT= mitochondrial aspartate aminotransferase; ASP TR=aspartate transporter.

bone during bone formation $[2,8]$. This is a specialized metabolic capability that does not typically (with some exception) occur with most normal functioning mammalian cells, which utilize citrate via the Krebs cycle for their bioenergetic/metabolic requirements (Fig. 1). Instead, net citrate production requires that mitochondrial citrate oxidation is inhibited so that the citrate can be exported to the cytosol and ultimately secreted out of the cell and into the extracellular environment (e.g. prostatic fluid for prostate cells; bone matrix for osteoblasts). Increased uptake and accumulation of zinc inhibits $\mathrm{m}$-aconitase and citrate oxidation in prostate cells. Since this removes 6-carbon citrate from the cell's metabolic pool, the continued production of citrate requires a 4-carbon source of oxalacetate along with the 2-carbon source of acetylCoA from glucose utilization. We identified aspartate as the 4carbon source for prostate cell citrate production [9].

In our earlier paper [2] we provided preliminary data that murine osteoblasts might exhibit the capability of citrate production. This present report with human osteoblasts and osteogenic mesenchyme cells now confirms and establishes for the first time that osteoblasts exhibit the metabolic relationships as specialized citrate-producing cells; and that this capability develops during the osteogenic differentiation of the mesenchyme cells.

\section{MATERIALS AND METHODS}

\section{Cell Culture}

Human mesenchymal stem cells (MSC) and osteoblasts were obtained from Lonza Group Ltd (hereafter referred to as "Lonza"). Mesenchyme cells were grown and maintained in Mesenchymal Cell Growth Supplement (MCGS, PT3001 ) with L-glutamine and containing $30 \mathrm{mg} / \mathrm{ml}$ gentamicin, $15 \mathrm{ug} / \mathrm{ml}$ amphotericin and $10 \%$ fetal bovine serum (FBS). Cells were cultured at $37{ }^{\circ} \mathrm{C}$ in the presence of
$5 \% \mathrm{CO}_{2}$ until $\sim 75 \%$ confluent. Mesenchyme cells were induced to differentiate to osteoblasts by culturing the cells in the presence of osteogenic medium from Lonza according to the manufacturer's protocol. Human primary osteoblasts were also obtained from Lonza. The primary osteoblasts were cultured in Clonetics Osteoblast Growth Medium especially formulated for the growth and expansion of human osteoblasts, and contained 10\% FBS, ascorbic acid and gentamicin-amphotericin.

\section{Citrate Production}

Citrate production by mesenchyme cells and osteoblasts was determined by culturing cells for 16 hours in DMEM supplemented with Chelex treated FBS (2\%), $50 \mu \mathrm{M}$ aspartate and $5 \mu \mathrm{M} \mathrm{ZnCl}_{2}$. Chelex treatment reduced the $\mathrm{Zn}$ concentration of the FBS to below the level of detection. After the 16 hour incubation with aspartate and $\mathrm{ZnCl}_{2}$ the medium was collected, deproteinized by addition of TCA to $7 \%$ and centrifugation at $3000 \mathrm{~g}$ for $5 \mathrm{~min}$. The citrate concentration of the media samples was measured by the fluoroenzymatic method previously described [10].

\section{Western Blot Analysis}

MSC and osteoblast cells were lysed using RIPA buffer (Upstate Biotech). The protein concentration of the lysates was determined using the BioRad protein assay based on the method of Bradford [11]. Proteins were separated by SDSPAGE and transferred to nitrocellulose membrane. The membranes were blocked by incubating in PBS containing $5 \%$ non-fat milk and $0.1 \%$ tween 20 for 2 hours at room temperature. ZIP1 was detected by incubating the membranes overnight with hZIP1 chicken polyclonal antibody as described previously [12]. Sodium coupled citrate transporter (NACT) protein was detected by incubating the membranes with NACT rabbit polyclonal antibody from Alpha Diagnostics overnight. hZIP1 and 
Table 1. Effect of physiological levels of zinc and aspartate on primary osteoblast citrate production.

\begin{tabular}{|c|c|}
\hline & Citrate Produced \\
\hline \hline DMEM & $(\mathrm{nmols} / \mathrm{ml})$ \\
\hline No substrate added & $73.0 \pm 15.7$ \\
\hline$+Z n(5 \mathrm{uM})+$ Asp $(50 \mathrm{uM})$ & $150.4 \pm 15.6^{*}$ \\
\hline
\end{tabular}

NACT protein bound antibody was detected by incubating the membranes with hydrogen peroxide labeled goat anti chicken or goat anti rabbit secondary antibody respectively and enhanced chemiluminescence detection reagents. The membranes were stripped and re-probed using anti $\beta$-actin antibody.

\section{siRNA ZIP1 Knockdown}

hZIP1 specific and non-target siRNA was obtained from Dharmacon RNAi Technologies and used to transfect cells. Primary osteoblasts were plated in 12 well plates in Lonza OMG medium supplemented with 10\% FBS and 1\% Penicillin-Streptomycin mixture and allowed to attach for 24 hours. After 24 hours of culture, the medium was changed and the cells overlaid with DharmaFECT 2 Transfection Reagent in OGM containing $1.0 \mu \mathrm{g} /$ well hZIP1 specific or non-target siRNA using the manufacturer's protocol. After 72 hours the non-target control and ZIP1 knockdown cells were washed with PBS and incubated with either no added substrate or with $50 \mu \mathrm{M}$ aspartate and $5 \mu \mathrm{M} \mathrm{ZnCl} \mathrm{Zn}_{2}$ in DMEM supplemented with $2.0 \%$ Chelex treated FBS for 1620 hours. After incubation with aspartate and $\mathrm{Zn}$ the medium was collected, deproteinized, and the cells lysed in RIPA buffer. The abundance of ZIP1 transporter was determined by Western blot analysis.

\section{Statistical Analysis}

The results were subjected to t-test analysis to determine statistical significance.

\section{RESULTS}

We proceeded with the expectation that the osteoblasts would likely exhibit the citrate-producing metabolic pathway similar to the pathway that we identified for prostate cells.
Table 2. Citrate production by mesenchyme cells and their differentiated osteoblasts.

\begin{tabular}{|c|c|}
\hline & Citrate $(\mathbf{n m o l s} \quad$ /ml) \\
\hline \hline Mesenchyme cells & $9.9 \pm 4.7$ \\
\hline Differentiated osteoblasts & $35.2 \pm 2.5^{*}$ \\
\hline
\end{tabular}

Cells maintained for 16 hours in DMEM+Zn+Asp

*Significant difference: $\mathrm{P}=0.009$

The major determinant would be a significant net production of citrate, which requires increased zinc uptake and accumulation to inhibit citrate oxidation as shown in Fig. (1). To determine this, cultured primary osteoblasts were exposed to either DMEM or DMEM supplemented with physiological levels of aspartate $(50 \mathrm{uM})$ and zinc $(5 \mathrm{uM})$ for 16 hours. Aspartate was included since it could be required for net citrate production as we identified for prostate cells (Fig. 1). Table 1 shows the significant $105 \%$ increase in citrate production in the presence of added physiological levels of aspartate and zinc.

In the following experiment, we determined if the capability of citrate production existed in osteoblasts that were differentiated from mesenchyme cells; and if this resulted from the differentiation of the mesenchyme cells, which are not citrate-producing cells. The mesenchyme cells were cultured in differentiation medium for 14 days when histological identification of osteoblast differentiation was evident as shown previously (Fig. 2) [13]. Then the differentiation medium was replaced with citrate-producing medium (DMEM+aspartate+zinc) as described in the preceding experiment. At that time, undifferentiated mesenchyme cells were also exposed to the citrate-producing medium. Table 2 shows that the mesenchyme cells produced minimal citrate as compared to the markedly significant increase of $\sim 250 \%$ in citrate production by the differentiated osteoblasts. We obtained similar preliminary results that we reported for the differentiation of the murine mesenchyme cells [2].

We further confirmed this relationship by determination of the expression of ZIP1 in the mesenchyme cells and the differentiated osteoblasts. ZIP1 is the important transporter for the uptake and accumulation of zinc in prostate cells (Fig. 1). In addition, ZIP1 upregulation during human mesenchyme cell osteogenic differentiation had been identified in an earlier report from our group (RBF coauthor

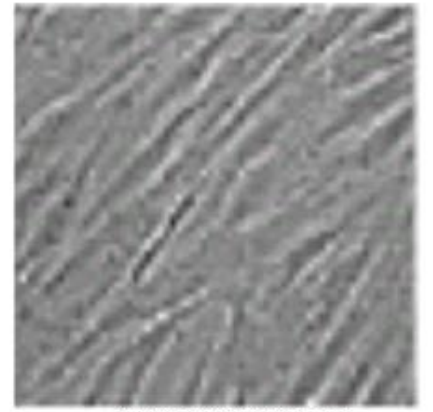

MESENCHYME CELLS

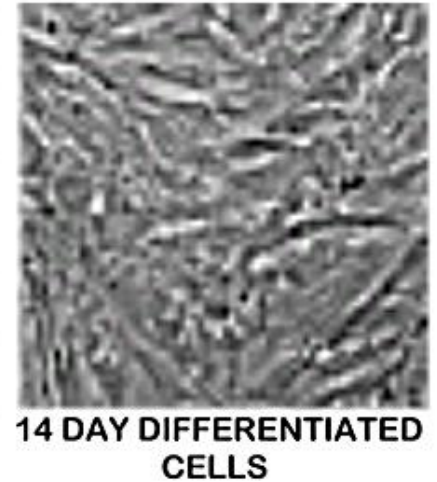

Fig. (2). Histological differentiation of mesenchymal cell to osteoblasts after 14 days culture in differentiation medium 


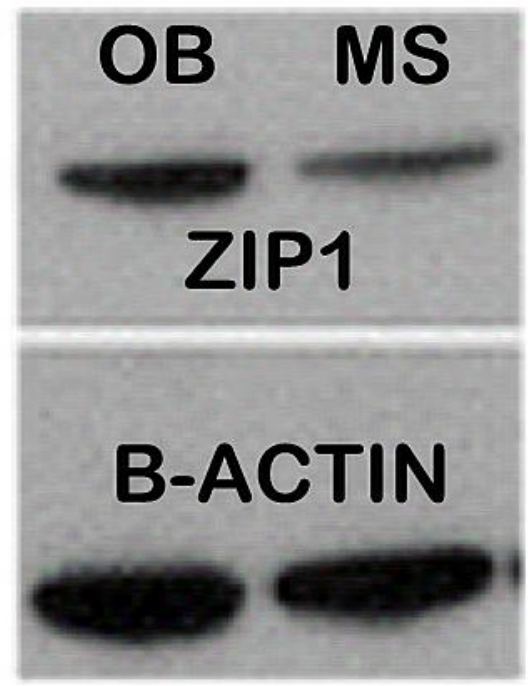

Fig. (3). Western blot analysis of ZIP1 abundance in mesenchyme cells versus differentiated osteoblasts.

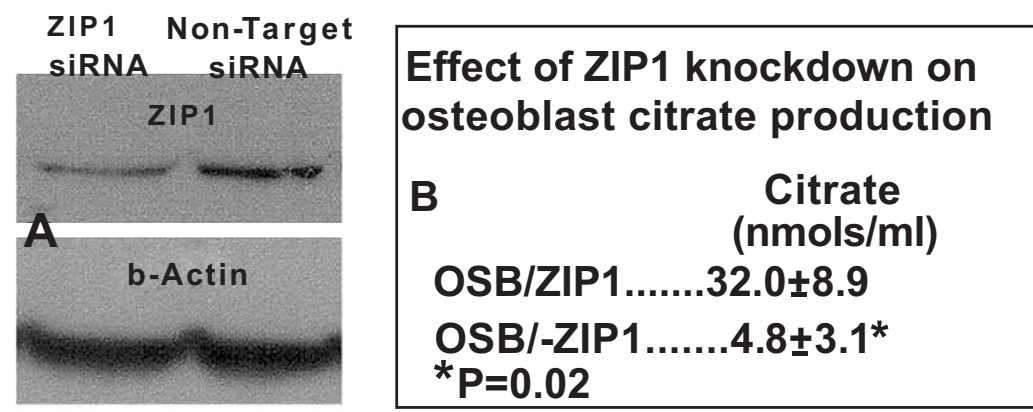

Fig. (4). Effect of ZIP1 knockdown on the production of citrate by primary osteoblasts. A. Western blot shows decreased ZIP1 transporter by ZIP1siRNA. B. Shows the loss of citrate production by ZIP1 knockdown.

[13]). Fig. (3) shows a relatively low abundance of ZIP1 transporter in the mesenchyme cells, and the markedly increased ZIP1 in the differentiated osteoblasts; and this is also evidence of the differentiation of the mesenchyme cells.

To confirm the importance of ZIP1 as the functional zinc uptake transporter required for osteoblast citrate production, we prepared primary osteoblasts with ZIP1 knockdown and determined the effect on citrate production. Fig. (4) shows the marked decrease in the abundance of ZIP1 transporter in the ZIP1 siRNA treatment. The knockdown of ZIP1 transporter resulted in a significant $\sim 85 \%$ decrease in osteoblast citrate production. This is also consistent with the earlier observation with human osteogenic mesenchyme cells [13] that ZIP1 upregulation is required for increased zinc uptake and osteoblast differentiation. Consequently, the identification of the requirement for upregulation of ZIP1 for citrate production by the osteoblasts is consist ent with the specialized functional metabolic capability of citrateproducing cells.

Whereas, our studies support the concept that osteoblast de novo synthesis and production of citrate is the major source of citrate for incorporation into bone, we could not dismiss the possibility that osteoblast transport of citrate from blood plasma might also be a source of citrate in bone. The plasma citrate concentration is $~ 110 \mathrm{uM}$; of which, at physiological $\mathrm{pH}, \sim 98 \%$ exists as the tricarboxyllic acid and $\sim 2 \%$ as the dicarboxyllic acid. Mammalian cells typically do not extract citrate from plasma; and to do so requires the upregulation of a plasma membrane citrate transporter. Under these conditions, the likely functional transporter would be the specific tricarboxyllate citrate transporter, NaCT (Slc13A5). Therefore we determined if this transporter is present in the primary osteoblasts. Since NaCT is highly expressed in liver cells [14], we employed HepG2 cells as a positive control. Western blot analysis (Fig. 5) revealed the absence of $\mathrm{NaCT}$ transporter in the osteoblast as compared to the abundant transporter protein in HepG2 cells. Thus, there remains no reported evidence that the citrate for bone formation is derived from osteoblasts transport of citrate from blood; and this further strengthens the importance of osteoblasts as citrate-producing cells.

\section{DISCUSSION}

This report, coupled with our earlier preliminary data [2] now establishes for the first time that osteoblasts are specialized citrate-producing cells. The study also establishes that the osteoblasts develop this specialized capability during the osteogenic differentiation of the mesenchyme cells since the latter are not citrate producing cells. It is also significant that we demonstrated that ZIP1 zinc uptake transporter is upregulated in the osteoblasts 


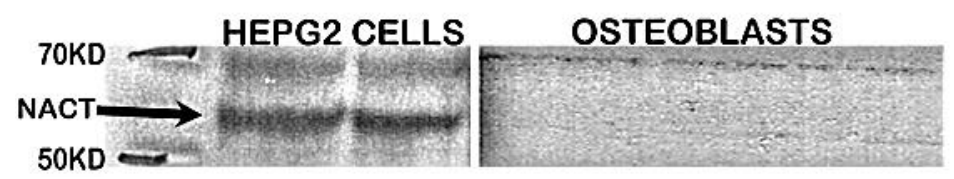

Fig. (5). Western blot of NaCT transporter abundance in osteoblasts versus HepG2 cells.

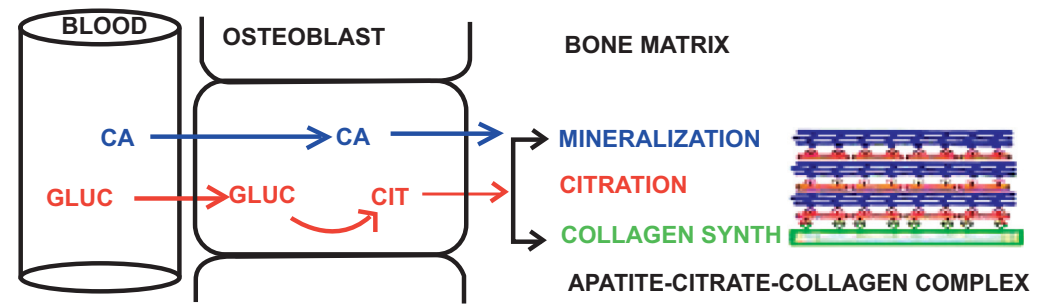

Fig. (6). Concept of the role of osteoblasts as specialized citrate-producing cells and the process of citration for the incorporation of citrate into the apatite/citrate/collagen complex during bone formation.

during differentiation, and that ZIP1 is essential for the production of citrate. It is well established that bone contains very high zinc levels; being the most abundant trace element in bone, and $\sim 10$ fold greater than found in most other tissues; and is implicated in bone disorders [15, 16]. However, among the various studies and speculations of the role of zinc in bone, the importance of zinc for osteoblast citrate production had not been considered in any of the reports aside from this study and our earlier reports $[2,8]$. It is becoming evident that citrate-producing cells, as represented by prostate cells and now osteoblasts, are also zinc accumulating cells; which is necessary to inhibit maconitase activity and citrate oxidation (Fig. 1) [17]. We are now proceeding to establish if aspartate is the essential 4carbon source for osteoblast net citrate production; and if the aspartate transporter EAAC1 is the important transporter as exists in the prostate cells (Fig. 1).

The results of this study address the important question, "What is the source of the citrate that is incorporated into the structure of bone?" The fact that citrate is a major component of bone in all vertebrates attests to the fact that it is an indispensible component that serves an essential structural/functional role; which had remained unknown. However, recent studies of $\mathrm{Hu}$ et al. [4-6] and Davies et al. [7] have identified that citrate is incorporated into the structure of the apatite nanocrystal/collagen complex, and is required to establish the optimal size of the nanocrystal structure in order to achieve the bone properties of stability, strength and resistance to fracture. Consistent with this, we have identified the presence of citrate in the mineral component of bone and in the apatite/collagen complex (unpublished information). Thus, it becomes evident that the availability and incorporation of citrate must be carefully regulated in concert with the mineralization process during the formation of the apatite nanocrystal/collagen complex. This relationship needs to be considered in the determination of the source of citrate for incorporation into bone during bone formation.

A long-held view has described bone citrate as being derived from blood plasma in combination with calcium transport. This is unlikely when one considers that the plasma citrate concentration is $\sim 110 \mathrm{uM}$ and the plasma calcium concentration is $\sim 2500 \mathrm{uM}$. A concurrent "transport" of a calcium-citrate complex from blood plasma would be limited by the low concentration of citrate. In addition, the necessary regulation and temporal integration and regulation of the citrate delivery for incorporation into the apatite structure in concert with mineralization would be unlikely. Also, the absence of detectable NaCT citrate transporter in the osteoblasts, and no reported evidence of osteoblast transport of citrate from blood, presently argue against plasma citrate as the source of citrate in bone.

In contrast, some early studies [18-20] demonstrated that bone preparations in vitro were capable of de novo synthesis and production of citrate; thereby indicating that bone cells might be the source of citrate in bone. However, the specific cells or metabolic pathway had never been identified. Our studies now demonstrate that the osteoblasts are citrateproducing cells. This supports our new concept (Fig. 6) that osteoblasts de novo synthesis and production of citrate provides the citrate that is incorporated into the apatite nanocrystal/collagen complex (which we refer to as "citration") during the process of bone formation. Consequently, the osteoblasts can be regulated to synchronize citration with its function of mineralization during the formation of the apatite nanocrystal/collagen complex; which now becomes an important issue that needs to be addressed.

This relationship also impacts the understanding of the implications of bone in the homeostatic regulation of the plasma citrate concentration; which is normally maintained at $\sim 110 \mathrm{uM}$. Since the skeleton contains $\sim 90 \%$ of the total body citrate, it has been considered to be the reservoir for citrate distribution and flux into and out of plasma. The view has considered that citrate along with calcium is taken up from circulation during bone formation; and it is released from bone along with calcium during bone resorption. This view purportedly was supported by the early observations that linked parathyroid hormone as a hypercalcemic/hypercitricemic agent; and calcitonin as a hypocalcemic/hypocitricemic agent. While this relationship exists for the release of calcium and citrate during bone resorption, it is becoming evident that it is not applicable regarding the source of citrate in bone. The newly identified 


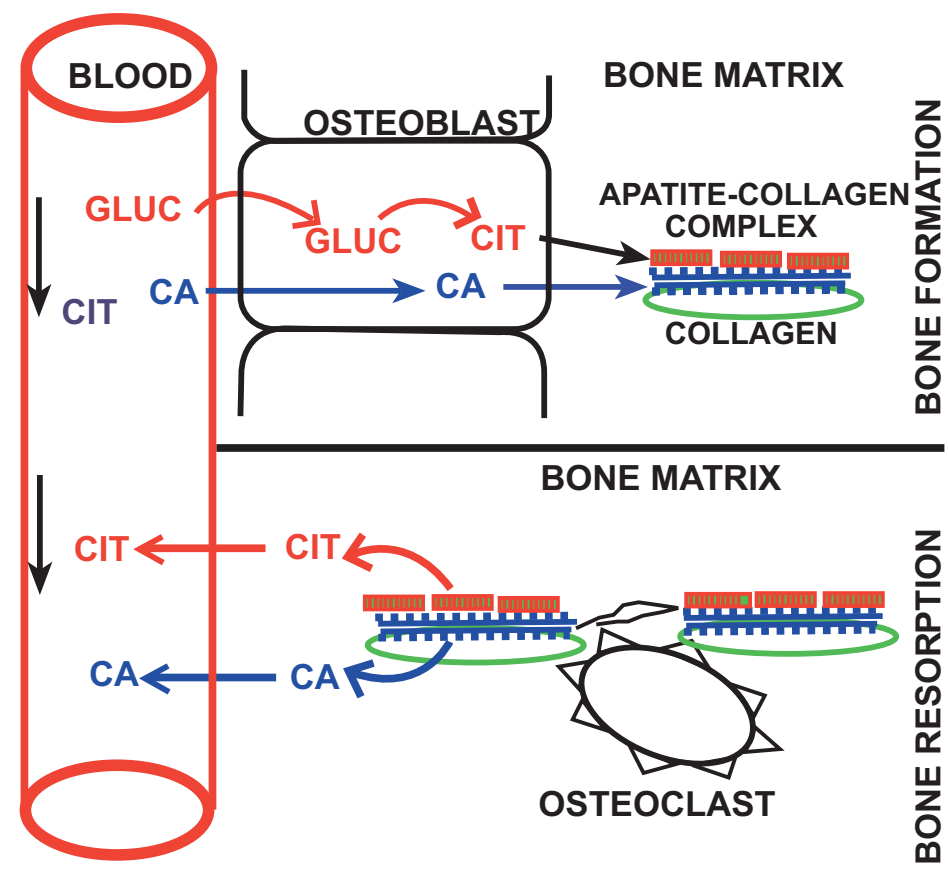

Fig. (7). Concept of the implications of bone citrate in the homeostatic maintenance of plasma citrate levels.

capability of the osteoblasts as citrate-producing cells along with the early reports that bone preparations were capable of de novo production of citrate now reveals that the osteoblasts provide an important source of de novo citrate for maintenance of plasma citrate levels. This provides a new understanding of the source of citrate in bone and the implications of bone in relation to plasma citrate (Fig. 7).

\section{CONCLUSION}

After nearly 75 years since the discovery of citrate as a major component of bone, we have arrived at the identification of osteoblast citrate production as the likely important source of citrate in bone. The understanding of bone formation must include the indispensible and essential importance of citrate incorporation into the structural component of bone; without which the bone will not exhibit its properties of stability, strength, and resistance to fracture. The process of bone formation involves the osteogenic transformation of mesenchyme cells to citrate-producing osteoblasts, which provide the source of citrate for incorporation into the bone structure (i.e. "citration") in concert with mineralization and the formation of the hydroxyapatite-citrate-collagen complex. This provides a new model for investigating and identifying the factors and conditions required to achieve normal bone formation. This new understanding has widespread implications in bone disorders and defects, in bone repair and regeneration, and in virtually all issues relating to bone formation. These observations also provide a new understanding of the implications of bone citrate in citrate homeostasis; in which osteoblast citrate production is an important source of de novo citrate for maintenance of plasma citrate levels. This will have impact on hormonal and other factors that regulate and maintain citrate homeostasis.

\section{CONFLICT OF INTEREST}

The authors confirm that this article content has no conflicts of interest.

\section{ACKNOWLEDGEMENTS}

This study was supported by NIH grant AR064808.

\section{REFERENCES}

[1] Dickens F. The citric acid content of animal tissues, with reference to its occurrence in bone and tumour. Biochem J 1941; 35: 101123.

[2] Costello LC, Franklin RB, Reynolds MA, Chellaiah M. The important role of osteoblasts and citrate production in bone formation: "osteoblast citration" as a new concept for an old relationship. Open Bone J 2012; 4: 27-34.

[3] Schwarcz HP, Agur K, Jantz LM. A new method for determination of postmortem interval: citrate content of bone. J Forensic Sci 2010; 55: 1516-22.

[4] Hu YY, Rawal A, Schmidt-Rohr K. Strongly bound citrate stabilizes the apatite nanocrystals in bone. Proc Natl Acad Sci U S A 2010; 107: 22425-9.

[5] Hu Y-Y, Liu XP, Ma X, et al. Biomimetic self-assembling copolymer_hydroxyapatite nanocomposites with the nanocrystal size controlled by citrate. Chem. Mater. 2011; 23: 2481-90.

[6] Schmidt-Rohr K. Citrate key in bone's nanostructure. ScienceDaily (June 8, 2011).

[7] Davies E, Müller KH, Wong WC, et al. Citrate bridges between mineral platelets in bone. Proc Natl Acad Sci USA 2014; 14: E1354-63.

[8] Costello LC, Franklin RB. A review of the important central role of altered citrate metabolism during the process of stem cell differentiation. J Regen Med Tissue Eng 2013; 2: 1-8.

[9] Franklin RB, Zou J, Yu Z, Costello LC. EAAC1 is expressed in rat and human prostate epithelial cells; functions as a high-affinity Laspartate transporter; and is regulated by prolactin and testosterone. BMC Biochem 2006; 27: 7:10.

[10] Costello LC, O'Neill JJ. A simplified and sensitive method for citrate determination in biological samples. J Appl Physiol 1969; 27: 120-2. 
[11] Bradford MM. A rapid and sensitive method for the quantitation of microgram quantities of protein untilizing the principle of proteindye binding. Anal Biochem 1976; 72: 248-54.

[12] Franklin RB, Ma J, Zou J, et al. Human ZIP1 is a major zinc uptake transporter for the accumulation of zinc in prostate cells. J Inorg Biochem 2003; 96: 435-42.

[13] Tang Z, Sahu SN, Khadeer MA, Bai G, Franklin RB, Gupta A. Overexpression of the ZIP1 zinc transporter induces an osteogenic phenotype in mesenchymal stem cells. Bone 2006; 38: 181-98.

[14] Inoue K, Zhuang L, Ganapathy V. Human Na+ -coupled citrate transporter: primary structure, genomic organization, and transport function. Biochem Biophys Res Commun 2002: 299: 465-71.

[15] Yamaguchi M. Role of nutritional zinc in the prevention of osteoporosis. Mol Cell Biochem 2010; 338: 241-54.
[16] Vidica-Gurban C, Mederle O. The OPG $\div$ RANKL system and zinc ions are promoters of bone remodeling by osteoblast proliferation in postmenopausal osteoporosis. Rom J Morphol Embryol 2011; 52: 1113-9.

[17] Costello LC, Liu Y, Franklin RB, Kennedy MC. Zinc inhibition of mitochondrial aconitase and its importance in citrate metabolism of prostate epithelial cells. J Biol Chem 1997; 46: 28875-81

[18] Kenny A, Draskczy P, Goldhaber P. Citric acid production by resorbing bone in tissue culture. Am J Physiol 1959; 197: 502-4.

[19] Dixon TF, Perkins HR. Citric acid and bone metabolism. Biochem J 1952; 52: 260-5.

[20] Seifter E, Lavine LS. Aspects of citric acid chemistry related to bone. Bull N Y Acad Med 1961; 37: 156-66.

(C) Franklin et al.; Licensee Bentham Open.

This is an open access article licensed under the terms of the Creative Commons Attribution Non-Commercial License (http://creativecommons.org/licenses/by$\mathrm{nc} / 3.0 /)$, which permits unrestricted, non-commercial use, distribution and reproduction in any medium, provided the work is properly cited. 